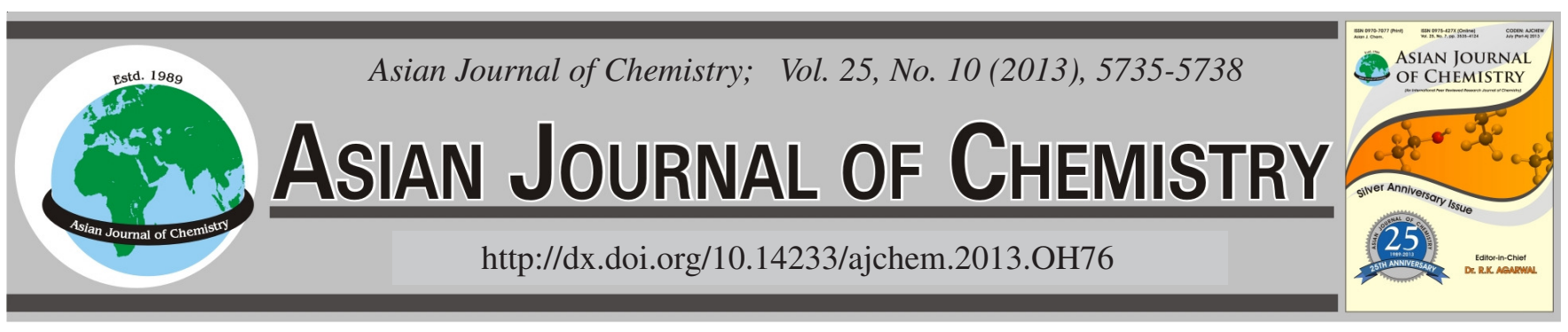

\title{
Characteristics of an Electrostatic Wiggler for Improved Free Electron Radiation $\dagger$
}

\author{
Dae-Wook Kim ${ }^{1}$, VinaYa Kumar Jha ${ }^{2}$, Seungjoon Ahn ${ }^{1}$, Tae-Sik OH ${ }^{1}$, Ho Seob Kim ${ }^{1}$ and Young Chul Kim ${ }^{3, *}$
}

${ }^{1}$ Department of Information Display, Sun Moon University, 100 Kalsanri, Tangjeong-myun, Asan-si, Chungnam 336-708, Republic of Korea ${ }^{2}$ Department of Physics and Nanoscience, Sun Moon University, 100 Kalsanri, Tangjeong-myun, Asan-si, Chungnam 336-708, Republic of Korea ${ }^{3}$ Department of Optometry, Eulji University, 553, Sanseong-daero, Sujeong-gu, Seongnam-si, Gyeonggi-do 461-713, Republic of Korea

*Corresponding author: Fax: +82 31 7407365; E-mail: yckim@eulji.ac.kr

\begin{abstract}
Characteristics and structure of a free electron radiation module composed of an electron source and an electrostatic wiggler have been investigated through a simulation, especially focused on a planar type wiggler. In order to improve the efficiency of planar type wiggler in asymmetric operation mode, a simple modification in the wiggler structure is suggested and the improved performance of the modified wiggler system will be discussed.
\end{abstract}

Key Words: Microcolumn, Electrostatic wiggler, Asymmetric mode, Electromagnetic radiation, Simulation.

\section{INTRODUCTION}

A free electron laser has attracted steady attention since it can generate electromagnetic waves with wide spectral range from microwave to ultraviolet and it is regarded to be very useful for various applications in near future ${ }^{1-4}$. In the free electron radiation, the radiation is generated from the motions of accelerated electrons due to a structure so called a wiggler. After the proposal of an electrostatic wiggler replacing the widely adopted magnetic undulator, steady effort has been devoted to develop electron radiation modules based on the electron source and the electrostatic wiggler for its convenient applications $^{5-12}$.

A miniaturized free electron radiation module is composed of a micro electron column and a wiggler. Generally, a miniaturized electron source called a microcolumn is composed of three parts ${ }^{13}$. The first part is electron source which emits and supplies electron beam (e-beam) to the other part of the microcolumn. The second part is a deflector and the last part is an Einzel lens playing the role of focusing e-beam. Since e-beam scanning or focusing is not necessary for a free electron radiation module, an electrostatic wiggler is attached to the first part of a microcolumn as schematically presented in Fig. 1. The electron source is composed of a sharp cold field emitter and three electrostatic lenses, so called source lens system; the first lens is to extract electrons from the emitter, the second one is an accelerator, and the third one is a limiting aperture used to limit the final e-beam diameter.

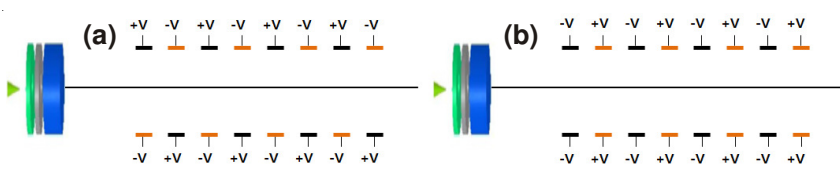

Fig. 1. Schematic diagram of free electron radiation module with planar type wiggler. (a) Asymmetric mode operation and (b) Symmetric mode operation

There are two types of electrostatic wiggler. One is the planar type and the other is ring type. For a planar type wiggler, normally opposite potentials are applied to the electrodes facing each other as schematically shown is Fig. 1(a) and this operation is called asymmetric mode. While the operation mode described in Fig. 1(b) is called symmetric mode in which the electrodes facing each other is biased with the same potential and opposite potential is applied to the next face-to-face electrode pair and so on. The periodically biased electrodes supply the oscillatory field, which will make electrons oscillate transversely and generate electromagnetic wave. And this periodicity determines the central wavelength of the wave $\mathrm{e}^{9,14}$.

In case of symmetric operation mode, e-beam shift is not severe and almost all of the e-beam entering the wiggler passes through the wiggler without hitting the electrodes. However, in this case, only the electrons within a limited range from the electrodes are expected to oscillate with large amplitude since the electrons propagating near the optical axis feel relatively weak transverse electric field, which is due to the bias configuration described in Fig. 1(b). 
The advantage of asymmetric mode operation is that relatively stronger electric field can be obtained than symmetric mode because of the bias configuration shown in Fig. 1(a). Therefore, almost all of the electrons passing the wiggler region are influenced by electric field and they oscillate with relatively large amplitude. However, when an electrostatic planer type wiggler is operated in asymmetric mode, the trajectories of electrons tend to shift toward one direction (Fig. 2), which is thought to be due to the strong influence of the first electrode pair. In other words, electrons feel the strong electric field from the first electrode pair as soon as they enter the wiggler and shift toward the positively biased electrode and this shift is so large that the repulsive field by the second electrode pair cannot sufficiently push back the electrons. Finally some of the electrons hit wiggler electrodes and cannot pass through the wiggler thoroughly. In this work, an improved design of a planar type wiggler to reduce the e-beam shift in asymmetric mode operation will be suggested and the details will be discussed accompanied by some simulation results.

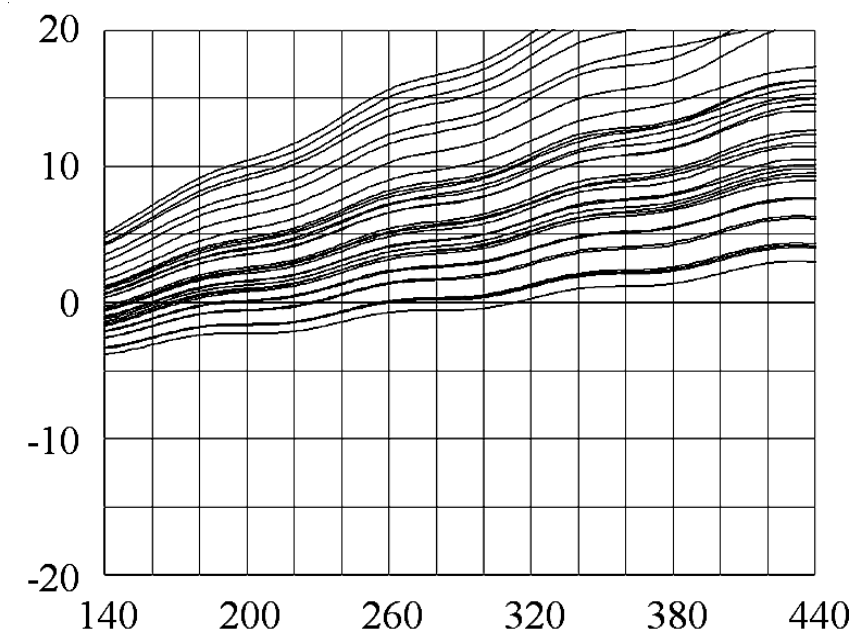

Fig. 2. Typical electron beam trajectories within a planar type wiggler operated in asymmetrical mode. The scale is in the unit of $\mu \mathrm{m}$

Improved design of a planar type wiggler: The deviation of the beam $(\Delta x)$ due to an electrode with width $\mathrm{W}$ is roughly proportional to the square of $\mathrm{W}$, i.e. $\Delta \mathrm{x} \propto \mathrm{W}^{2}$. We tried to modify the wiggler structure by making the width of the first electrode (w) of a wiggler narrower than those of other normal electrodes (W) so that e-beam deviation due to the first electrode is reduced. The narrow electrode will result in the reduction of overall e-beam shift in a wiggler system since the influence of the first electrode is restricted by the relation $\Delta \mathrm{x} \propto \mathrm{w}^{2}$.

Fig. 3(a) and (b) illustrate the difference between the commonly used wiggler and the design with narrow first electrode, respectively. In order to demonstrate the effect of the first electrode narrower than the other electrodes, a series of free electron radiation modules having different $\mathrm{W}$ are designed and we tried to investigate the e-beam trajectories in each model. It is noticed that the electrodes at both ends in this figure are not biased but grounded in order to keep the wiggler in a condition isolated from the external influences such as the potential or field due to the source part of the microcolumn.

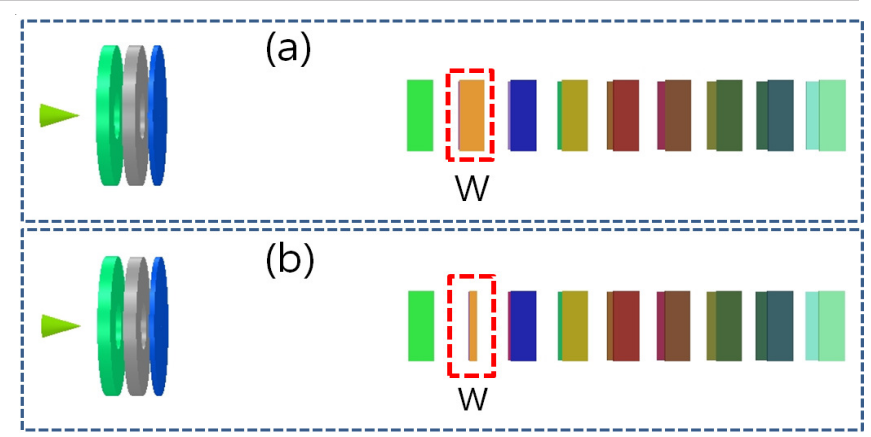

Fig. 3. Modified design of a planar type wiggler. (a) The width of the first electrode is same as those of other electrodes, W. (b) The width of the first electrode $(\mathrm{w})$ is narrower than $\mathrm{W}$

The structure of the wiggler studied in this work is basically same as that described in Fig. 1(a) or Fig. 3(a), where the width of all electrodes (W) are $10 \mu \mathrm{m}$ and the gap between electrodes are also $10 \mu \mathrm{m}$. And the space between two faceto-face electrodes is $40 \mu \mathrm{m}$ [Fig. 4(a)]. In a modified model, $\mathrm{w}$ is reduced keeping the other parameters fixed as in Fig. 3(b). We selected total 4 models with different values of $w$, i.e., $\mathrm{w}=10,7,3$ and $1 \mu \mathrm{m}$, for model $1,2,3$, and 4 , correspondingly.

For the beam extraction, a negative voltage was applied to the emitter $\left(\mathrm{V}_{\text {Tip }}=-300 \mathrm{~V}\right)$ and the first (extractor) and the third (limiting aperture) electrostatic lens of the microcolumn source part are grounded. We applied an appropriate negative voltage (in this case $-240 \mathrm{~V}$ ) to the second electrostatic lens for the purpose of making e-beam parallel to the optical axis or slightly converging, which will maximize the beam current passing through the wiggler. To the electrodes of wiggler, bias potential of $\pm 50 \mathrm{~V}$ or $\pm 100 \mathrm{~V}$ are applied to supply the oscillating transverse field.

\section{RESULTS AND DISCUSSION}

In Fig. 4, the simulation results obtained from two free electron radiation modules, ordinary and modified wigglers are presented. The width of the first electrode (w) in Fig. 4(a) and (b) are $\mathrm{w}=10 \mu \mathrm{m}$ and $\mathrm{w}=1 \mu \mathrm{m}$, that is model 1 and 4 , respectively. Except w, other parameters are fixed and same each other. As one can see clearly, e-beam shifts severely in Fig. 4(a) while the shift is considerably reduced in Fig. 4(b), which indicates that the modified wiggler design is effective for the reduction of beam shift.

In order to analyze electron trajectory and the effect of $\mathrm{w}$, we reduced the aperture diameter of the limiting aperture (the third electrostatic lens of the microcolumn source part)
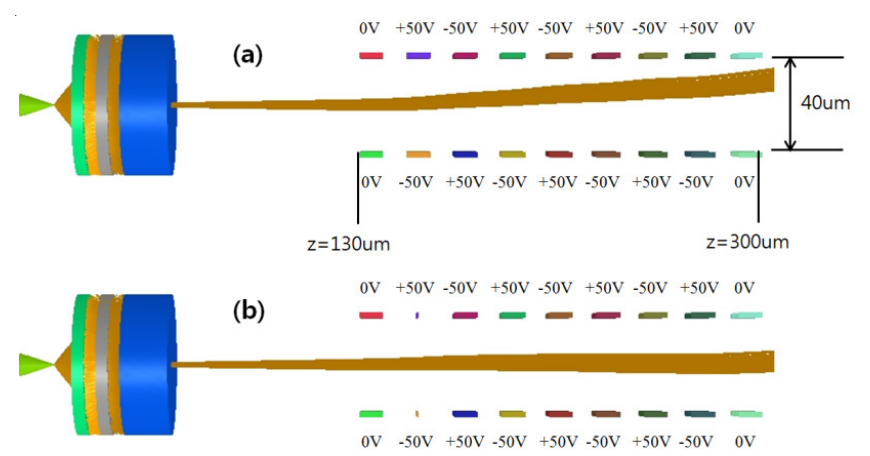


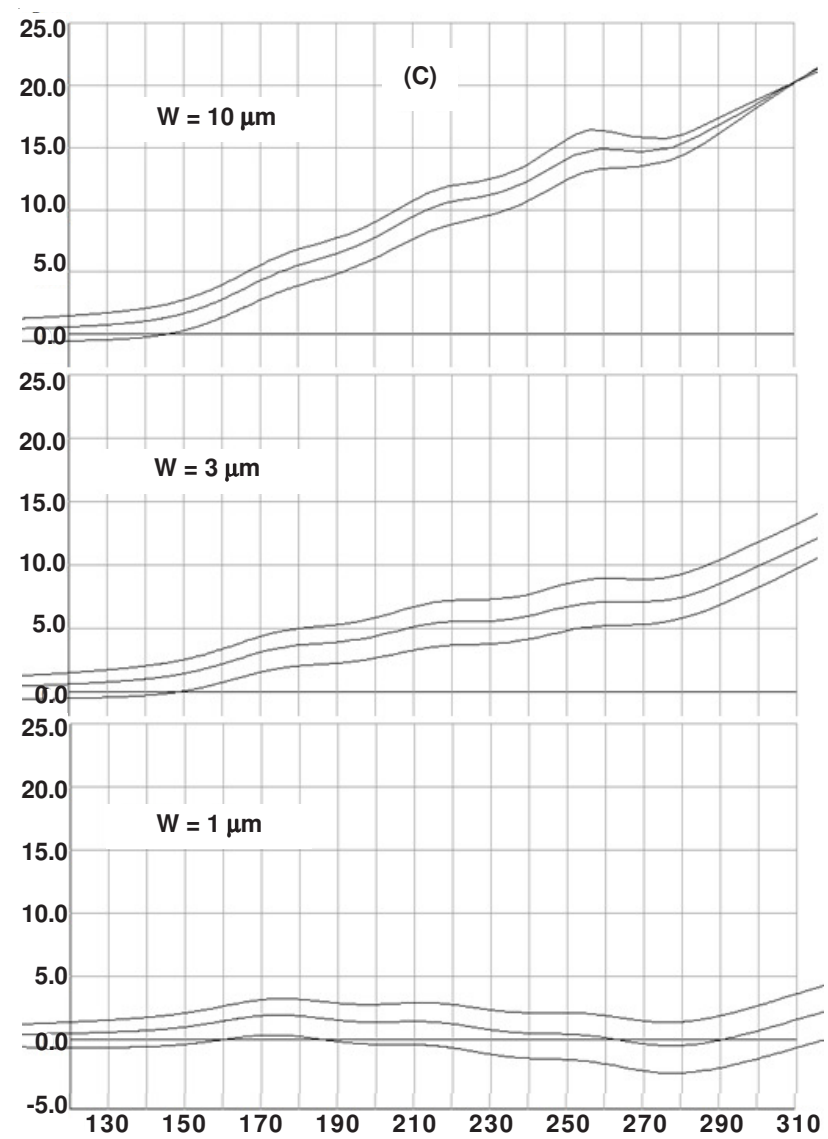

Fig. 4. Comparison of the simulation results obtained from two electrostatic wigglers with different first electrode width, w, when wiggler bias voltage is $\pm 50 \mathrm{~V}$. (a) $\mathrm{w}=10 \mu \mathrm{m}$ and (b) $\mathrm{w}=1 \mu \mathrm{m}$. (c) In this case, the diameter of limiting aperture is reduced down to $0.4 \mu \mathrm{m}$ and the wiggler bias voltage is $\pm 100 \mathrm{~V}$

extremely small, $0.4 \mu \mathrm{m}$, and repeated similar simulation for the 4 models with different values of $w$. The simulation results obtained when the wiggler bias voltage was fixed at $\pm 100 \mathrm{~V}$ are presented in Fig. 4(c). It is clear that the shift of electron trajectory becomes smaller with decreasing $\mathrm{w}$ and the beam propagate through the wiggler nearly parallel to the optical axis when $w=1 \mu \mathrm{m}$ (model 4). Simulation performed with wiggler bias voltage of $\pm 50 \mathrm{~V}$ showed similar features, and the results are summarized in Fig. 5.

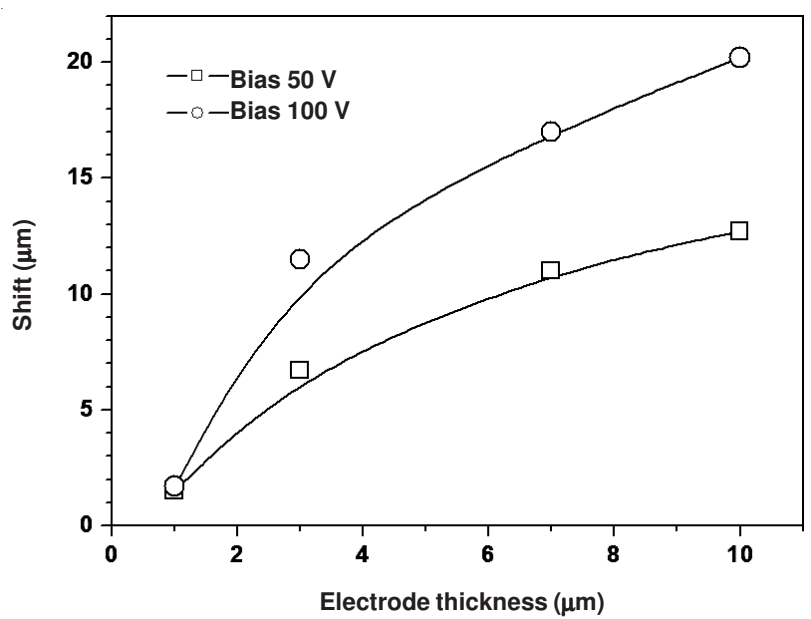

Fig. 5. Variation of e-beam shift depending on the width of the first electrode
The graphs in Fig. 5 present the variations of e-beam shift depending on w. Open circles and squares represent the shifts when bias voltage is $\pm 50 \mathrm{~V}$ and $\pm 100 \mathrm{~V}$, respectively. Regardless of the bias voltage, the beam shift becomes smaller as w decreases and finally the shift becomes negligible (around $1 \mu \mathrm{m}$ ) for $\mathrm{w}=\mathrm{W} / 10$ case, i.e., model 4 . This means that we can increase the number of wiggler period enough by extending the wiggler length, which will increase the radiation intensity.

To understand the basic mechanism of shift reduction, the variations of potential and electric field inside the wiggler system have been systematically analyzed. As an example, the variation of electric field inside the wiggler system is presented in Fig. 6. The open circles and solid squares represent data from the model 1 and $4(\mathrm{w}=10 \mu \mathrm{m}$ and $\mathrm{w}=1 \mu \mathrm{m})$, respectively. In this case, the data was obtained along $\mathrm{z}$-axis when $\mathrm{r}=5 \mu \mathrm{m}$ apart from the optical axis as shown in Fig. 6.

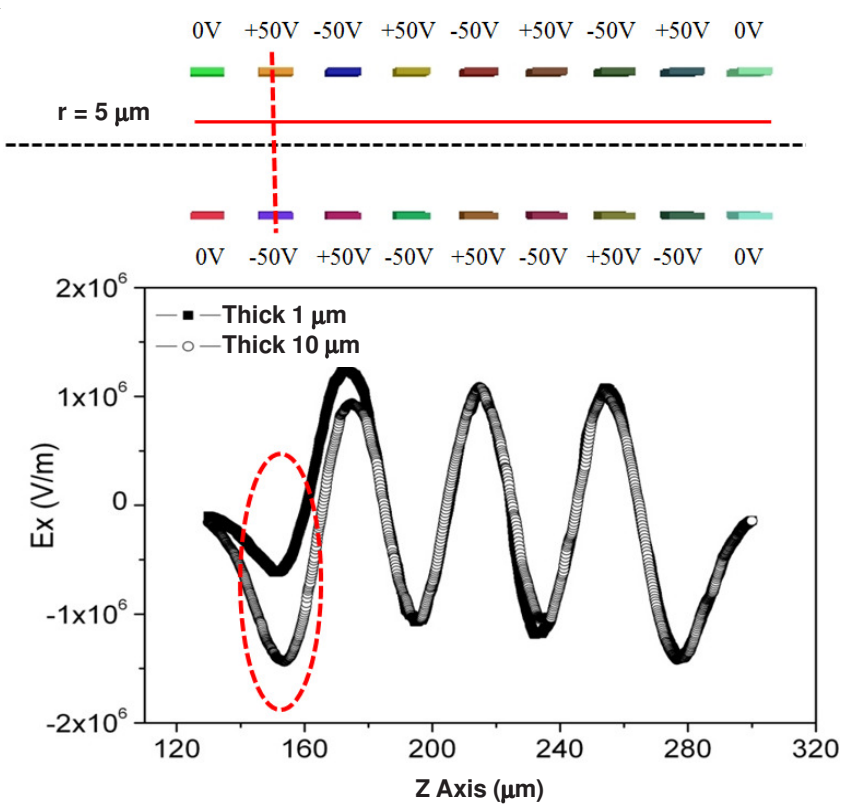

Fig. 6. Electric field variations inside the wiggler along $\mathrm{z}$-axis when $\mathrm{r}=5 \mu \mathrm{m}$ apart from the optical axis

For both open circles and solid squares, overall trend of oscillation is same as expected by the periodicity of the wiggler bias. The difference appeared at the position of the first electrode is denoted by the dotted circle in Fig. 6. The field strength due to narrow electrode (model $4, \mathrm{w}=1 \mu \mathrm{m}$, solid squares) is much weaker than that of wider electrode (model $1, \mathrm{w}=10 \mu \mathrm{m}$, open circles) at the entrance of the wiggler. Therefore, the force applied to electrons due to electric field is smaller in case of model 4 than that in model 1, which eventually results in the smaller shift in model 4.

Although we performed simulation work selecting a few values of $\mathrm{w}$ for a specific value of $\mathrm{W}$, this argument can be generalized to an arbitrary value of $\mathrm{w}$ if the relation between $\mathrm{w}$ and $\mathrm{W}$ follows same relation. Thus, this preliminary work suggests that the beam shift, one of the main constraints in a planar type electrostatic wiggler, can be overcome by introducing a narrower electrode at the entrance region of the wiggler. 


\section{Conclusion}

Characteristics of a planar type electrostatic wiggler have been investigated in order to improve the efficiency of a free electron radiation module combined with a micro electron column. The performance of a planar type electrostatic wiggler operated in asymmetric mode can be improved by controlling the width of the electrode at the entrance region, which is expected to be superior to the normal case as well as the symmetric mode operation. According to our preliminary results, the e-beam shift can be simply overcome by adopting a narrower electrode and this will eliminate some restrictions in designing the optimized free electron radiation module.

\section{ACKNOWLEDGEMENTS}

This work was supported by the Sun Moon University Grant of 2012.

\section{REFERENCES}

1. G. Gallot, S.P. Jamison, R.W. McGowan and D. Grischkowsky, J. Opt Soc. Am., B17, 851 (2000).
2. K. Kawase, J. Shikata and H. Ito, J. Phys. D: Appl. Phys., 35, R1 (2002).

3. M. Nagel, P.H. Bolivar, M. Brucherseifer, H. Kurz, A. Bosserhoff and R. Buttner, Appl. Phys. Lett., 80, 154 (2002).

4. S. Kumar, B.S. Williams, S. Kohen, Q. Hu and J.L. Reno, Appl. Phys. Lett., 84, 2494 (2004).

5. H. Motz, J. Appl. Phys., 22, 527 (1951).

6. G. Bekefi and R.E. Shefer, J. Appl. Phys., 50, 5158 (1979).

7. A. Anselmo and J.A. Nation, IEEE Trans. Nucl. Sci., 32, 3494 (1985).

8. S. Riyopoulos, Phys. Rev., E56, 4710 (1997).

9. A. Murai, K. Mima, S. Kuruma, N. Ohigashi, Y. Tunawaki, K. Imasaki, S. Nakai and C. Yamanaka, Nucl. Instrum. Methods Phys. Res. A, 331, 680 (1993).

10. V.A. Papadichev, Nucl. Instrum. Methods Phys. Res. A, 393, 403 (1997).

11. Y.C. Kim, S.J. Ahn, H.S. Kim, D.W. Kim and S. Ahn, Nucl. Instrum. Methods Phys. Res. A, 654, 427 (2011).

12. F. Floreani, H.W. Koops and W. Elsäber, Nucl. Instrum. Methods Phys. Res. A, 483, 488 (2002).

13. E. Kratschmer, H.S. Kim, M.G.R. Thomson, K.Y. Lee, S.A. Rishton, M.L. Yu, S. Zolgharnain, B.W. Hussey and T.H.P. Chang, J. Vac. Sci. Technol. $B, \mathbf{1 4}, 3792$ (1996).

14. S.J. Smith and E.M. Purcell, Phys. Rev., 92, 1069 (1953). 